\title{
Endoscopic submucosal dissection for nonampullary large superficial adenocarcinoma/adenoma of the duodenum: feasibility and long-term outcomes
}

Authors

Institutions
Shu Hoteya ${ }^{1}$, Naohisa Yahagi ${ }^{1,2}$, Toshiro lizuka' ${ }^{1}$, Daisuke Kikuchi' ${ }^{1}$, Toshifumi Mitani ${ }^{1}$, Akira Matsui ${ }^{1}$, Osamu Ogawa', Satoshi Yamashita' ${ }^{1}$, Tsukasa Furuhata ${ }^{1}$, Akihiro Yamada ${ }^{1}$, Ryusuke Kimura ${ }^{1}$, Kosuke Nomura $^{1}$, Yasutaka Kuribayashi ${ }^{1}$, Mitsuru Kaise ${ }^{1}$

${ }^{1}$ Department of Gastroenterology, Toranomon Hospital, Tokyo, Japan

${ }^{2}$ Cancer Center, Keio University, Tokyo, Japan

\section{Bibliography}

Dol http://dx.doi.org/

10.1055/s-0033-1359232

Endoscopy International Open

2013; 1: E2-E7

(c) Georg Thieme Verlag KC

Stuttgart · New York

E-ISSN 2196-9736

Corresponding author:

Shu Hoteya, MD, PhD

Department of

gastroenterology, Toranomon

Hospital,

2-2-2 Toranomon, Minato-ku,

Tokyo 105-8470,

Japan

torashu@hotmail.com
Background and study aims: The clinical benefit of endoscopic submucosal dissection (ESD) for superficial duodenal neoplasias has not yet been verified. The aims of this study were to validate the feasibility and long-term outcomes of ESD for nonampullary superficial duodenal neoplasias, larger than $20 \mathrm{~mm}$.

Patients and methods: 41 patients, with 41 nonampullary large superficial duodenal neoplasias, who underwent ESD between April 2005 to March 2013 were included in the study. The short- and long-term outcomes were retrospectively evaluated, related to tumor size, resection size, histological type, invasion depth, complete resection rate, operation time, perforation rate, delayed bleeding rate, local recurrence rate, distant metastasis, and survival rate.

Results: ESD was successfully completed in 38 of 41 patients (92.7\%). The median sizes of tumors and resected specimens were, respectively, 26

\section{Introduction}

Superficial duodenal neoplasias are rare and their clinical characteristics are unknown. Endoscopic resection of duodenal neoplasias has been performed in a small number of cases, but an adequate management strategy for these tumors remains undefined because of their rarity and unclear clinical behavior.

Small duodenal adenomas are resectable en bloc by conventional endoscopic mucosal resection (EMR) [1 -7]. However, the therapeutic indication for small benign neoplasias is uncertain because their malignant potential is obscure and a watchand-wait strategy is a valid option. In contrast, local resection is required for large duodenal adenomas and superficial carcinomas because they may have malignant potential for progression. However, curative resection of these lesions with a malignant nature is fairly difficult using conven- $\mathrm{mm}$ (range $20-70 \mathrm{~mm}$ ) and $36.5 \mathrm{~mm}$ (range 23$80 \mathrm{~mm}$ ). Histopathological findings showed 13 adenocarcinomas (12 mucosal, 1 submucosal) and 28 adenomas (26 high grade dysplasia, 2 low grade dysplasia). The complete resection rate was 89.5\% (34/38 tumors). The mean operation time was 128.3 minutes. Perforation during ESD occurred in $39 \%$ and delayed bleeding occurred in 18.4\%. The median observation period was 48 months (range 3.2-94.1 months), and no local recurrence or distant metastasis was seen during this time, representing a disease-free survival rate of $100 \%$

Conclusions: ESD for nonampullary large superficial duodenal adenocarcinoma/adenoma is feasible with favorable long-term outcomes. However, because of the high complication rate due to its technical difficulty, experience and skill of the operator, along with adequate perioperative management, are essential.

tional EMR, therefore endoscopic submucosal resection (ESD) is a compelling alternative.

ESD is well established as a promising procedure that is both curative and safe even in large lesions, and is accepted as a minimally invasive therapy for superficial neoplasias of the stomach, esophagus, and colon [8-10]. In contrast, duodenal ESD is technically challenging because of anatomical specificities, and has not been accepted as a radical method of local resection to date.

This study aimed to validate the feasibility and long-term outcomes of ESD applied to duodenal superficial neoplasias, larger than $20 \mathrm{~mm}$, which are outside the indication for conventional EMR. 


\section{Patients and methods}

\section{V}

\section{Patients}

A total of 63 superficial nonampullary duodenal tumors in 63 consecutive patients (46 men and 17 women; mean age 61.5 years) were resected by ESD at Toranomon Hospital between April 2005 and March 2013. Out of the 63 patients, we analyzed 41 patients with superficial duodenal tumors larger than $20 \mathrm{~mm}$ in diameter, excluding 22 patients with tumors less than $20 \mathrm{~mm}$ in diameter.

Pretreatment indications for ESD were a noninvasive adenocarcinoma or a large adenoma that was suspected of having a cancerous component, based on endoscopic findings and pathological findings from biopsy specimens. Endoscopic ultrasonography was performed using a UM-2 R system (Olympus Optical, Tokyo, Japan) when submucosal invasion was suspected. All patients provided their written, informed consent for the proposed procedure, and surgical resection was offered as an option before treatment.

\section{ESD procedure}

The ESD procedure was performed using a Dual Knife or Flex Knife and/or Hook Knife (KD-650, KD-630L, KD-620; Olympus Optical) through a two-channel multi-bending gastroscope and another conventional gastroscope with waterjet functions (GIF2TQ260 M, GIF-Q260J; Olympus Optical) and a $\mathrm{CO}_{2}$ insufflation system (UCR; Olympus Optical), as previously reported for gastric ESD $[8,11]$. When the gastroscope was not long enough to remove the lesion, a colonoscope was used (PCF-Q260JL/I; Olympus Optical).

In brief, a soft transparent hood (D-201-13404; Olympus Optical, or DH-15GR; Fujifilm Medical) was attached to the tip of the gastroscope to obtain good, direct, gastroscopic views of the submucosal layer. As the margin of the duodenal tumor could be detected clearly by chromoendoscopy using indigo carmine dye spraying, marking dots were not usually necessary. We used a high frequency electrosurgical generator model ICC200 or VIO300 D (ERBE Elektromedizin, Tubingen, Germany). Depending on the model used, the electrosurgical unit was set at a cutting current on Endocut mode, effect 2, 40W (ICC200) or Drycut mode, effect 2, 40W (VIO300D) for mucosal incision, and at a coagulating current for submucosal dissection on Forced Coagulation mode, 40W (ICC200) or Swift Coagulation mode, effect 4, $40 \mathrm{~W}$ (VIO300D). After submucosal injection of glycerol (10\% glycerol and 5\% fructose; Chugai Pharmaceutical, Tokyo, Japan), containing $0.005 \%$ indigo carmine and $0.0025 \%$ epinephrine, a mucosal incision was made outside the tumor margin. Hyaluronic acid was added to the injection solution when mucosal elevation was insufficient. After the mucosal incision, direct dissection of the submucosal layer was performed to obtain the optimal specimen, and a complete en bloc resection could be achieved. After removal of the lesion, some visible vessels located in the bottom of the ulcer were treated with hemostatic forceps (HDB2422W; Pentax, Tokyo, Japan, or Coagrasper, FD-410LR; Olympus Optical) or clips (EZ clip, HX110LR; Olympus Optical) for prevention of delayed bleeding. Finally, sucralfate solution was sprayed onto the ulcer to protect it from digestive juices.

\section{Perioperative management}

Despite the fact that ESD for esophageal, gastric, and colorectal tumors is usually conducted with the patient under conscious sedation, most of the duodenal ESD procedures were performed with the patient under general anesthesia with endotracheal intubation in an operating theatre, if the procedure time was expected to exceed 2 hours. Stable general anesthesia can relieve some of the technical difficulties associated with duodenal ESD, such as poor endoscopic maneuverability in the narrow lumen, stressful submucosal dissection of the thin wall, and an unstable endoscopic view during respiratory fluctuations or intestinal spasm.

If perforation happened during the procedure, the defect was closed by endoscopic clipping or sutured by endoloop (HX21L1, MAJ339; Olympus Optical) in some cases, followed by the administration of antibiotics and fasting for several days. Abdominal computed tomography was performed to confirm the retroperitoneal perforation in all cases of perforation including suspicious cases. If closure of the perforation was not achieved and ESD could not be completed, we converted from ESD to open surgery without hesitation.

Postoperative delayed bleeding was defined as hematemesis or melena which needed an endoscopic hemostatic procedure during a second endoscopy and using hemostatic forceps or clips, similar to the preventive procedure described above. We performed a second endoscopy systematically in all patients in order to decide when to start oral intake. All patients received a proton pump inhibitor (PPI) for at least 2 months after ESD. Follow-up endoscopic examinations were performed after 2 and 6 months, and then every 12 months. Abdominal computed tomography or ultrasonography was performed annually or more frequently to identify lymph node and distant metastases if final pathological findings of the resected specimen showed cancer.

\section{Histopathological evaluation}

The resected specimen was cut into 2-mm thick slices after formalin fixation. Histological type, size, depth of invasion, lateral and vertical margins, and lymphovascular invasion were evaluated in each slice. In this study, the criteria for declaring complete resection were en bloc resection with lateral and vertical margins free from tumor. Incomplete resection was defined as the performance of piecemeal resection or a histologically positive specimen margin.

\section{Clinical outcomes}

To verify the technical feasibility of duodenal ESD, we analyzed the following short-term outcomes: tumor size, resection size, histological type, invasion depth, complete resection rate, operation time, perforation rate, and delayed bleeding rate. To validate curability using duodenal ESD, we evaluated the following longterm outcomes: local recurrence rate, distant metastases and disease-free survival rate. Data are presented as median (range) or mean (standard deviation [SD]).

\section{Results}

\section{$\nabla$}

- Table 1 shows the clinicopathological characteristics of the 41 lesions removed from the 41 patients ( 29 men, 12 women), who had a mean (SD) age of 60.7 (9.0) years (range 34-77 years). Two-thirds of the tumors ( 26 of $41 ; 63.4 \%$ ) were located in the second portion of the duodenum, and the majority were flat elevated type by macroscopic appearance (36 of 41 ; $87.8 \%$ ). Histopathology of resected specimens showed 13 adenocarcinomas (12 mucosal, 1 submucosal) and 28 adenomas (26 high grade dysplasia, 2 low grade dysplasia). 
Table 1 Clinicopathological characteristics of 41 lesions in 41 patients with duodenal tumors larger than $20 \mathrm{~mm}$ who underwent duodenal ESD

\begin{tabular}{|ll|}
\hline Sex (male/female), $n$ & $29 / 12$ \\
\hline Age (y), mean \pm SD & $60.7 \pm 9.0$ \\
\hline Range & $(34-77)$ \\
\hline Tumor location, $n$ & \\
\hline First portion (anterior/posterior) & $4 / 2$ \\
\hline SDA (inner curve/outer curve) & $6 / 1$ \\
\hline Second portion (inner curve/outer curve) & $7 / 19$ \\
\hline IDA inside & 1 \\
\hline Third portion posterior & 1 \\
\hline Macroscopic findings, $n$ & \\
\hline Protruded/flat elevated & $3 / 36$ \\
\hline Depressed & 2 \\
\hline Preoperative biopsy finding, $n$ & 16 \\
\hline No biopsy & 17 \\
\hline Low-grade dysplasia & 5 \\
\hline High-grade dysplasia & 3 \\
\hline Adenocarcinoma & $13(12 / 1)$ \\
\hline Histology, $n$ & 26 \\
\hline Adenocarcinoma (M/SM) & 2 \\
\hline Adenoma with high-grade dysplasia & \\
\hline Adenoma with low-grade dysplasia &
\end{tabular}

Abbreviations: ESD, endoscopic submucosal dissection; IDA, inferior duodenal angle; $\mathrm{M}$, intramucosal invasion; SD, standard deviation; SDA, superior duodenal angle; SM, submucosal invasion.

\section{Short-term outcomes}

Of the 41 patients enrolled, 3 patients (7\%) required immediate surgical conversion because of uncontrollable perforation during the ESD procedure ( $\boldsymbol{O}$ Table 2 ). All 3 tumors were removed by ESD and local closure or drainage was performed by open surgery. Consequently, ESD was completed without surgical conversion in 38 of 41 patients, giving a success rate of $92.7 \%$.

- Table 3 shows the short-term outcomes relating to the 38 lesions in the 38 patients who were treated successfully by ESD. The median size of tumor and resected specimen were $26 \mathrm{~mm}$ (range $20-70 \mathrm{~mm}$ ) and $36.5 \mathrm{~mm}$ (range $23-80 \mathrm{~mm}$ ), respectively. ESD was performed with general anesthesia in 29 of these 38 patients. In most cases, general anesthesia was required based on expected procedure time because of various factors, such as scope maneuverability, tumor size, location, abundant vessels, lumen narrowness, and spasms. Perforation during ESD occurred in 12 of 38 patients (31.6\%) and was closed endoscopically by clipping. Ten patients showed only retroperitoneal emphysema without abscess development and 2 patients showed only peritoneal emphysema without abscess development. Delayed bleeding occurred in 7 of 38 patients (18.4\%). All patients with delayed bleeding were managed safely and successfully by coagulation using hemostatic forceps or by clipping. Thus both these complications were managed successfully by conservative treatment. Delayed perforation occurred at the site of ESD the next day in 1

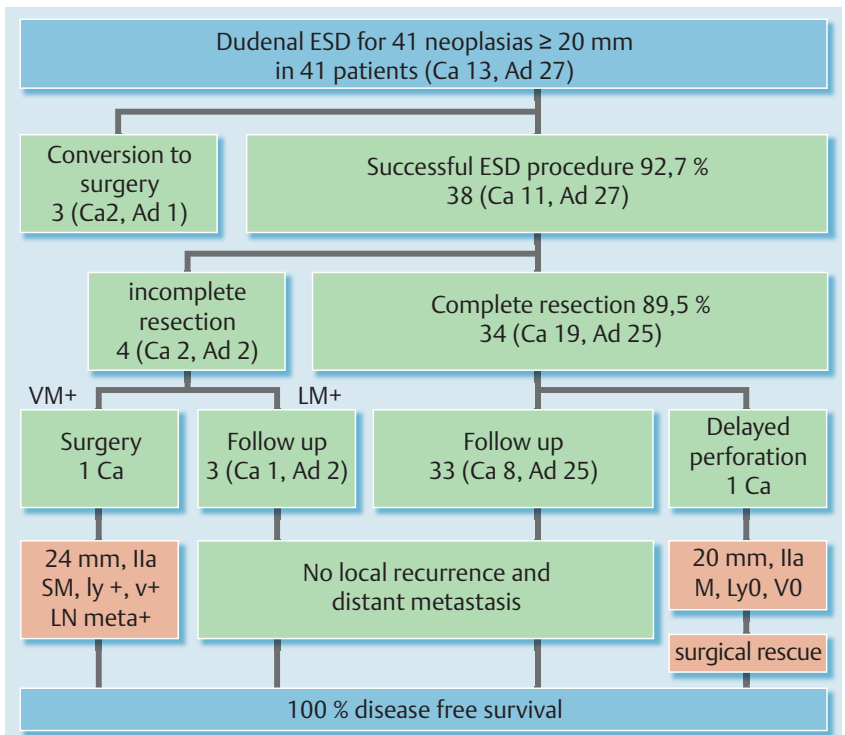

ESD, endoscopic submucosal dissection; Ca, carcinoma; Ad, adenoma; VM+, vertical margin positive; $\mathrm{LM}+$, lateral margin positive; SM, submucosal invasion; $\mathrm{M}$, intramucosal; ly, lymphatic invasion; v, vascular invasion; LN meta, lymph node metastasis

Fig. 1 Flow chart of the short-term and long-term outcomes of duodenal endoscopic submucosal dissection (ESD).

patient, who required local closure and drainage of an abscess by open surgery 5 days after ESD. All 38 patients were discharged after a mean (SD) hospitalization of 10.3 (4.2) days (range 6-26 days). After discharge from the hospital, no further complications including secondary stenosis occurred.

- Fig. 1 demonstrates the clinical course of the 38 successful ESD procedures, and a representative case of duodenal ESD is shown in $\bullet$ Fig. 2.

The complete resection rate was $89.5 \%$ (34 of 38 tumors). Three patients with positive lateral margins (one adenocarcinoma and two adenomas) were followed without additional treatment with a median observation period of 52 months (range, 49.388.2 months). One patient was positive for a deep margin with positive lymphovascular and submucosal invasion of adenocarcinoma. By previous endoscopic ultrasonography, submucosal invasion was suspected but no lymph node metastasis was detected. This patient received an additional curative pancreaticoduodenectomy, and subsequent histopathology revealed local lymph node metastasis.

\section{Long-term outcomes}

All 37 patients who completed ESD were followed according to the post-ESD observation protocol. The median observation period was 48 months and ranged from 3.2 to 94.1 months. No local recurrence or distant metastasis was demonstrated during

Table 2 Characteristics of three patients who switched to surgery due to uncontrollable perforation

\begin{tabular}{|c|c|c|c|c|c|c|c|c|}
\hline $\begin{array}{l}\text { Age } \\
\text { (years)/sex }\end{array}$ & $\begin{array}{l}\text { Duodenal } \\
\text { location }\end{array}$ & $\begin{array}{l}\text { Size } \\
(\mathrm{mm})\end{array}$ & $\begin{array}{l}\text { Macroscopic } \\
\text { type }\end{array}$ & Final diagnosis & Depth & $\begin{array}{l}\text { Lymphovascular } \\
\text { invasion }\end{array}$ & $\begin{array}{l}\text { Hospitalization } \\
\text { (d) }\end{array}$ & $\begin{array}{l}\text { Prognosis } \\
\text { (mo) }\end{array}$ \\
\hline $62 / F$ & $\begin{array}{l}\text { Second, outer } \\
\text { curve }\end{array}$ & 23 & II a & High-grade adenoma & M & Negative & 14 & DFS 51.3 \\
\hline $63 / M$ & Third posterior & 27 & II a & Adenocarcinoma & M & Negative & 17 & DFS 13.4 \\
\hline $64 / F$ & $\begin{array}{l}\text { Second, outer } \\
\text { curve }\end{array}$ & 36 & II a & Adenocarcinoma & M & Negative & 25 & DFS 83.3 \\
\hline
\end{tabular}

Abbreviations: DFS, disease-free survival; M, intramucosal invasion. 

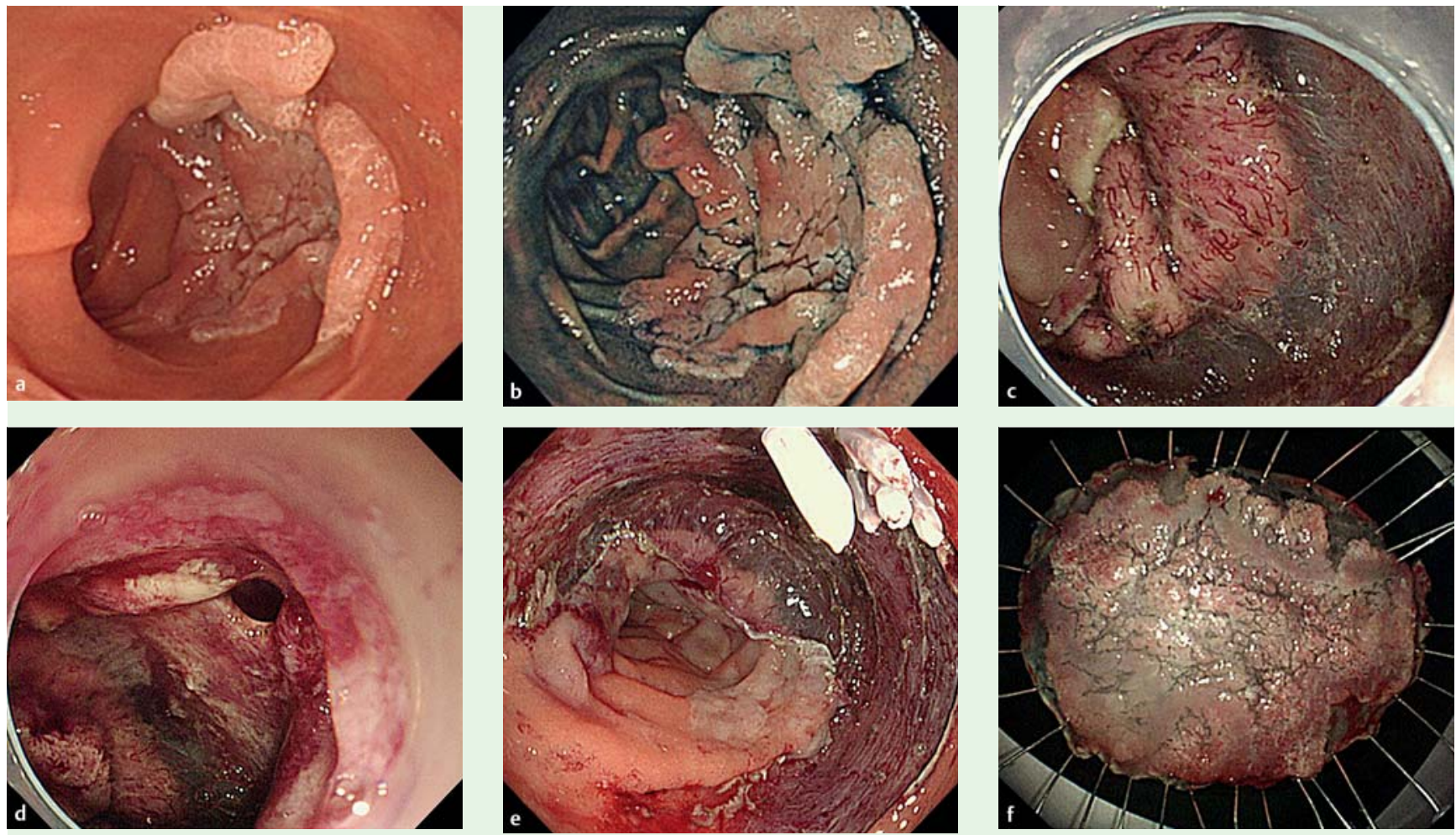

Fig.2 A representative case of duodenal endoscopic submucosal dissection (ESD). a A large superficial neoplasm occupying two-thirds of the descending portion of the duodenum. $\mathbf{b}$ Chromoendoscopy finding after indigo carmine dye spraying. $\mathbf{c}$ Abundant blood vessels were observed in the submucosal layer. d A perforation occurred during the ESD procedure. e The perforation was closed by endoscopic clipping and successfully managed conservatively. $\mathbf{f}$ Resected specimen: $57 \times 44 \mathrm{~mm}$, well-differentiated adenocarcinoma with adenomatous component, depth intramucosal, no lymphatic invasion (ly0), no vascular invasion (v0), margin-negative.

Table 3 Outcomes of complete ESD procedure for 38 lesions in 38 patients

\begin{tabular}{|ll}
\hline Tumor size (mm), median & 26 \\
\hline (Range) & $(20-70)$ \\
\hline Resected specimen size $(\mathrm{mm})$, median & 36.5 \\
\hline (Range) & $(23-80)$ \\
\hline General anesthesia/conscious sedation, $n$ & $29 / 9$ \\
\hline Operation time $(\mathrm{min})$, mean \pm SD & $128.3 \pm 69.5$ \\
\hline Perforation rate, $\%(n)$ & $31.6(12)$ \\
\hline Delayed perforation rate, \% $(n)$ & $2.6(1)$ \\
\hline Delayed bleeding rate, \% $(n)$ & $18.4(7)$ \\
\hline Hospitalization (day), mean \pm SD & $10.3 \pm 4.2$ \\
\hline Complete resection ${ }^{1}$ rate, \% $(n)$ & $89.4(34)$ \\
\hline Incomplete resection rate, \% $(n)$ & $11.6(4)$ \\
\hline Lateral/vertical positive, $n$ & $3 / 1$ \\
\hline Observation periods (months), median & 48 \\
\hline (Range) & $(3.2-94.1)$ \\
\hline Local recurrence, $n$ & 0 \\
\hline Distant metastasis, $n$ & 0 \\
\hline Prognosis, DFS rate, \% $(n)$ & $100(38)$ \\
\hline
\end{tabular}

Abbreviations: DFS, disease-free survival; ESD, endoscopic submucosal dissection.

1 En bloc resection with lateral and vertical margin free from tumor. this period, resulting in a disease-free survival rate of $100 \%$ ( Table 3).

\section{Discussion}

$\nabla$

Superficial neoplasias of the duodenum are rare, and the outcome of duodenal endoscopic resection, especially that of ESD, is not well known. Three studies reported the short-term outcomes of duodenal ESD in a small number of cases $(<10)$ [12-14], and another reported 14 ESD cases with adenoma and a mean tumor size of $17 \mathrm{~mm}$ [15]. To our knowledge, the present study is the first to evaluate the short- and long-term outcomes of ESD in a substantial number of patients with superficial duodenal neoplasias larger than $20 \mathrm{~mm}$, and a mean tumor size of $32 \mathrm{~mm}$. These large duodenal tumors are not amenable to conventional EMR, because en bloc resection is rarely accomplished and a substantial number of local recurrences, even in small tumors, are reported using EMR [3-6]. In contrast, our data demonstrate that ESD can achieve an acceptable completion rate (93\%) and R0 resection rate (89\%). No local recurrence and a disease-free survival rate of $100 \%$ for the median 48 months of the observation period indicate the potential of ESD for curing large superficial tumors of the duodenum.

In spite of the high rate of cure by duodenal ESD shown here, perforation occurred in one-third of the patients (39\%) and conversion to surgery due to uncontrollable perforation was undertaken in 4 of 41 patients (9.7\%), in 3 perioperatively and in 1 with delayed perforation 1 day after ESD who required surgery 5 days after ESD for local closure and abscess drainage. Our perforation 
rate is comparable to the perforation rate of $31 \%$ (10 of 32 patients) described in the four previously mentioned publications [12-15], indicating that duodenal ESD is still a challenging procedure compared with ESD in the esophagus, stomach, and colon, where perforation rates are reportedly low [9-11]. The duodenum has specific anatomical features that make the ESD procedure difficult. The narrow and more deeply located lumen forces an endoscopic maneuver that makes it difficult to maintain an adequate visual field during the procedure. In addition, the duodenal wall is the thinnest in the gastrointestinal tract. The submucosal layer, where Brunner's glands are abundant, is densely fibrous in a nonpathological state. Thus, a submucosal injection, even using a hyaluronic acid solution, barely elevates the mucosa enough for safe mucosal cutting and submucosal dissection. Taken together, these anatomical features may cause a higher rate of perforation during duodenal ESD. However, because of the variety of factors related to perforations, as described above, it is difficult to clarify the main factors likely to cause them. However, all our perforation cases, other than the 3 surgical conversions, were successfully managed in a conservative manner. Intensive care during and after ESD is essential to prevent serious perforationrelated complications; in addition, we performed supplementary treatments, such as endoclip closure of perforations and administration of PPI and antibiotics.

We experienced 3 instances of perforations (7\%) that needed immediate surgical conversion, which is now rarely carried out during ESD of the gastrointestinal tract other than for the duodenum. Since we conducted those duodenal ESDs with patients under general anesthesia in an operating theatre, we could smoothly convert to surgery and all 3 patients were discharged with only 1 week of additional hospitalization (mean 17 days), compared with patients without surgery. Nevertheless, the rate of surgical conversion is not negligible, and thus meticulous cooperation with surgeons is essential for a favorable rescue and clinical course. Actually, various factors are related to successful ESD, which are difficult to predict before ESD, and often change during the ESD procedure. Therefore, we concluded from limited experience that general anesthesia is absolutely necessary to provide better conditions, and for immediate surgical conversion, if needed.

Delayed bleeding occurred at a rate of $18 \%$, which is considerably higher than after gastric, esophageal or colonic ESD. Abundant blood vessels in the submucosal layer and exposure of the ESD ulcer to bile acid and pancreatic juice may cause delayed bleeding. Prophylactic coagulation of visible vessels in the ESD ulcer bed immediately after the resection is known to be effective in reducing post-ESD bleeding. However, we perform prophylactic coagulation at the minimum level, because excess coagulation may induce incurable damage to the thin duodenal wall and may result in delayed perforation. Therefore, post-ESD management is crucial, including a sufficient duration of fasting, PPI administration, or a second endoscopy for deciding when to start oral intake. However, the actual timing of the second endoscopy varied, because we decided the timing based on various factors, such as tumor location, resected size, excess bleeding during the procedure, and comorbidities. Further studies are required to clarify the benefit and optimal timing of a second endoscopy.

Clinical questions about the behavior of superficial duodenal neoplasias remain unanswered $[1,2,16]$ : How fast does low grade adenoma progress to invasive cancer? How frequently does submucosal cancer metastasize? At least, our present data showed that superficial neoplasias larger than $20 \mathrm{~mm}$ in diame- ter might have malignant potential; one-third and two-thirds of these tumors were mucosal carcinomas and high grade adenomas, respectively, and included a submucosal cancer which metastasized to the local lymph node. Therefore, R0 local resection is necessary as much for an appropriate pathological evaluation as for treatment. No distant metastasis was observed in the 13 patients with mucosal carcinoma in the present study. Previous reports have shown that the incidence of lymph node metastasis is $0 \%$ in cases of mucosal cancer [6,17-19]. Taking these findings together, mucosal carcinoma of the duodenum is a good indication for ESD. Additional surgery in the case of submucosal cancer, especially with positive lymphovascular invasion, might be considered, but further studies are required to address this issue. In conclusion, the present study validates the feasibility of ESD for large nonampullary superficial duodenal adenocarcinoma/adenoma, although its technical difficulty necessitates much greater skill, compared with ESD in other sites of the gastrointestinal tract. Since complication rates were relatively high, adequate perioperative management including thorough cooperation with the surgeon is essential for obtaining good clinical outcomes. Further multicenter studies need to establish the appropriate therapeutic strategy based on a reliable diagnosis for superficial duodenal neoplasias.

\section{Competing interests: None.}

\section{References}

1 Obata S, Suenaga M, Araki $K$ et al. Use of strip biopsy in a case of early duodenal cancer. Endoscopy 1992; 24: 232 - 234

2 Oka S, Tanaka S, Nagata $S$ et al. Clinicopathologic features and endoscopic resection of early primary nonampullary duodenal carcinoma. J Clin Gastroenterol 2003; 37: 381 - 386

3 Apel D, Jakobs R, Spiethoff $A$ et al. Follow-up after endoscopic snare resection of duodenal adenomas. Endoscopy 2005; 37: 444-448

4 Lepilliez V, Chemaly M, Ponchon $T$ et al. Endoscopic resection of sporadic duodenal adenomas: an efficient technique with a substantial risk of delayed bleeding. Endoscopy 2008; 40: 806-810

5 Alexander S, Bourke MJ, Williams SJ et al. EMR of large, sessile, sporadic nonampullary duodenal adenomas: technical aspects and long-term outcome (with videos). Gastrointest Endosc 2009; 69: 66-73

6 Abbass R, Rigaux J, Al-Kawas FH. Nonampullary duodenal polyps: characteristics and endoscopic management. Gastrointest Endosc 2010; 71: $754-759$

7 Sohn JW, Jeon SW, Cho CM et al. Endoscopic resection of duodenal neoplasms: a single-center study. Surg Endosc 2010; 24: 3195-3200

8 Yahagi $N$, Fujishiro M, Kakushima $N$ et al. Endoscopic submucosal dissection for early gastric cancer using the tip of an electrosurgical snare (thin type). Dig Endosc 2004; 16: 34-38

9 Oyama T, Tomori A, Hotta $K$ et al. Endoscopic submucosal dissection of early esophageal cancer. Clin Gastroenterol Hepatol 2005; 3: 67 - 70

10 Yamamoto $H$, Yahagi $N$, Oyama T. Mucosectomy in the colon with endoscopic submucosal dissection. Endoscopy 2005; 37: 764-768

11 Hoteya S, Iizuka T, Kikuchi D et al. Benefits of endoscopic submucosal dissection according to size and location of gastric neoplasm, compared with conventional mucosal resection. J Gastroenterol Hepatol 2009

12 Honda T, Yamamoto H, Osawa $H$ et al. Endoscopic submucosal dissection for superficial duodenal neoplasms. Dig Endosc 2009; 21: 270274

13 Takahashi T, Ando T, Kabeshima $Y$ et al. Borderline cases between benignancy and malignancy of the duodenum diagnosed successfully by endoscopic submucosal dissection. Scand J Gastroenterol 2009; 44: $1377-1383$

14 Endo M, Abiko Y, Oana $S$ et al. Usefulness of endoscopic treatment for duodenal adenoma. Dig Endosc 2010; 22: 360-365

15 Jung JH, Choi KD, Ahn JY et al. Endoscopic submucosal dissection for sessile, nonampullary duodenal adenomas. Endoscopy 2013; 45: $133-135$ 
16 Hirasawa $R$, Iishi $H$, Tatsuta $M$ et al. Clinicopathologic features and endoscopic resection of duodenal adenocarcinomas and adenomas with the submucosal saline injection technique. Gastrointest Endosc 1997; 46: 507-513

17 Kim HK, Chung WC, Lee BI et al. Efficacy and long-term outcome of endoscopic treatment of sporadic nonampullary duodenal adenoma. Gut Liver 2010; 4: 373-377
18 Basford PJ, Bhandari P. Endoscopic management of nonampullary duodenal polyps. Therap Adv Gastroenterol 2012; 5: 127-138

19 Poultsides GA, Huang LC, Cameron JL et al. Duodenal adenocarcinoma: clinicopathologic analysis and implications for treatment. Ann Surg Oncol 2012; 19: 1928 - 1935 